\title{
Factors Associated With Low Self-Esteem Among Patients With Hematologic Malignancies: A Cross-Sectional Study
}

\author{
Junye $\mathrm{Yu}^{1}$, Huan Dong ${ }^{2}$, Qi Wu ${ }^{2}$, Ya Yang ${ }^{2}$ and Hongying $\mathrm{Pi}^{1 *}$ \\ ${ }^{1}$ Chinese PLA General Hospital, Beijing, China, ${ }^{2}$ Aerospace Center Hospital, Beijing, China
}

The present study aimed to explore the self-esteem level of patients with hematology malignancies and the associated factors. In this cross-sectional study, we screened patients with hematologic malignancies treated in the Department of Hematology of Aerospace Center Hospital between November 2019 and June 2020. We included 157 eligible patients by convenience sampling. The participants completed questionnaires covering demographic characteristics, loneliness, the coping styles, hope, five personality traits, and self-esteem level. We applied $t$-test, analysis of variance, simple linear regression, and multiple stepwise regression to explore the factors associated with patients' self-esteem. The mean self-esteem score of hematology tumor patients was $26.86 \pm 2.34$, which was lower than the national norm $(P<$ 0.01). The multiple stepwise regression analysis identified maintaining close relationships with others, conscientiousness, extroversion, agreeableness, and positive coping style as factors independently associated with the self-esteem of hematology tumor patients. Patients with hematologic malignancies experience low self-esteem. Factors including maintaining close relationships with others, conscientiousness, extraversion, agreeableness, and active coping style were found to be independently associated with the self-esteem level of these patients. For patients with hematologic malignancies, health providers should apply patient-centered and targeted interventions to improve patients' self-esteem, reduce adverse psychological emotions, and improve their quality of life.

Specialty section: This article was submitted to Public Mental Health, a section of the journal

Frontiers in Psychiatry

Received: 30 March 2021

Accepted: 29 June 2021

Published: 19 August 2021

Citation:

Yu J, Dong H, Wu Q, Yang Y and Pi H (2021) Factors Associated With Low

Self-Esteem Among Patients With Hematologic Malignancies: A

Cross-Sectional Study. Front. Psychiatry 12:683894. doi: 10.3389/fpsyt.2021.683894

Keywords: hematology malignancies, cancer, self-esteem, associated factors, coping style, personality

\section{INTRODUCTION}

Hematologic malignancies are malignant clonal diseases originating from hematopoietic tissue and have become the fourth most common malignant tumor worldwide $(1,2)$. This disease is also among the top 10 high-incidence tumors in China, with leukemia being the 9th leading cause of cancer-related deaths after significant increases in its incidence $(3,4)$.

Due to the progression of the disease and anti-neoplastic treatments, patients with hematological malignancies always experience significant symptoms, including hair loss, vomiting, fatigue, insomnia, dizziness, pain, and even neuropathy (5). Additionally, the stigma of cancer and the despair caused by the prognosis can place great psychological pressure on patients and lead to psychological complications (6). All of the above factors can seriously affect the diagnosis, treatment, and prognosis of the disease, as well as the patient's mood, beliefs, and behavior (7-9). 
Self-esteem, as an important sentiment in Chinese native culture (10), is an evaluative and emotional experience of an individual to oneself and represents our sense of self-worth (11). Self-esteem can be threatened by the consequences of various diseases, but high self-esteem is an important prerequisite for successful coping (12). People with high self-esteem have good adaptability (13). However, changes in body image among patients with hematologic malignancies due to the side effects of radiotherapy and chemotherapy can cause low self-esteem (14). Low self-esteem is likely to lead to depression, which can have a negative impact on patients' physical and mental health $(13,15)$. Studies on self-esteem among patients with hematologic malignancies outside of China have been reported (16-20). A descriptive correlation study reported that Iranian leukemia patients' level of hope was positively correlated with self-esteem $(n=85)$, and appropriate education plans were designed and implemented to help increase hope and self-esteem in leukemia patients (5). However, there is a lack of studies investigating the self-esteem of patients with hematologic tumors in China. Therefore, the present study aimed to explore the self-esteem levels of Chinese patients with hematology malignancies and the associated factors to provide a reference for the development of further targeted measures to improve patients' quality of life.

\section{METHODS}

The present study was a cross-sectional study. The Ethics Committee of the Space Center Hospital approved the study (approval \#20200108-BSYJS-01). After receiving all study information, each patient signed a consent form before enrollment in this study. This study was reported following the requirements of the STrengthening the Reporting of OBservational studies in Epidemiology (STROBE) statement (21).

\section{Participants}

For this study, we screened patients with various types of hematological malignancies who were hospitalized in the Aerospace Center Hospital between November 2019 and June 2020. The inclusion criteria were: (1) age $>16$ years, (2) diagnosis with malignant blood disease, (3) stable condition, (4) knowledge of their disease diagnosis, (5) ability to understand and respond to the questionnaire questions, and (6) willingness to participate in this study. The exclusion criteria included: the presence of other severe physical illnesses and mental illness or cognitive impairment.

\section{Sample Size Calculation}

We applied convenience sampling for the questionnaire survey. We calculated the sample size needed for the present crosssectional study using the following formula:n $=\left(\frac{Z_{1-\alpha / 2} \times \sigma}{\delta}\right)^{2}$ (22). In this formula, $\mathrm{n}$ is the desired sample size; $\mathrm{Z}_{1-\alpha / 2}$ is a standard value for the corresponding level of confidence; $\sigma$ is standard deviation; $\delta$ is tolerance error. We applied 1.96 to $\mathrm{Z}_{1-\alpha / 2}$ at a $95 \%$ confidence interval (CI). We set a tolerance error $(\delta)$ of 0.8 , and the standard deviation $(\sigma)$ was $4.86(23)$. The sample size $(n)$ required was calculated to be 141 . Considering a $10 \%$ dropout rate of study participants, 156 hospitalized patients diagnosed with hematologic malignancies were needed.

\section{Data Collection}

After the research assistants obtained the consent of the patients' physicians, a specially trained investigator was responsible for the direction and management of the questionnaire. After the patients or their caregivers signed the informed consent form, the investigator distributed the questionnaire to the patients oneon-one. The investigator explained the purpose, significance, and completion method of the questionnaires to the patients and their families and provided unified instruction to briefly explain the scale. In principle, all questionnaires should have been completed by the patient independently within about $30 \mathrm{~min}$. For patients who could not fill in their answers independently, the investigator could assist them in completing the questionnaires item-by-item without suggestion or guidance. After the completion of the scale, the investigator would check and collect the questionnaires on the spot. If any missing or questionable issues were found, the response would be refilled or verified immediately to ensure the authenticity of the questionnaires.

The basic clinical characteristics of the patients were collected using a pre-tested questionnaire designed by the researchers. The income level in this study was a self-evaluation by patients according to average local income levels supplied in the questionnaire. Family visit refers to the number of family visits during hospitalization. Generally, more family visits reflect more concern by the family about the patient. The other independent variables included the patients' conditions (including the loneliness experienced), coping styles, hope level, and personality traits. The main outcome was the level of patients' self-esteem.

The loneliness experienced was measured using the University of California, Los Angeles Loneliness Scale as revised by Russell et al., which has 20 items, including nine inverse questions (24). The four-point Likert scale ( 1 = "never," 2 = "rarely," 3 = "sometimes" 4 = "always") was adopted with a total score of 20-80 points; a higher score reflected a stronger loneliness experience. The Cronbach's coefficient $\alpha$ for the scale was 0.84 , and the test-retest reliability was 0.62 .

A person's coping style is their typical manner of confronting and dealing with a stressful situation, and this was evaluated among the patients using the Simplified Coping Style Questionnaire (SCSQ) $(25,26)$. The SCSQ includes a total of 20 items and is mainly used to assess the attitudes and practices of individuals when they encounter difficulties or setbacks. The scale uses a four-point Likert scale method, from "not taken coping" to "often taken coping" in order, corresponding to 0 to 3 points. The Cronbach's $\alpha$ coefficient for the total scale is 0.90 , and the Cronbach's $\alpha$ coefficients for the Positive Coping Style Scale and the Negative Coping Style Scale are 0.89 and 0.78 , respectively, which indicates good reliability and validity. A higher positive coping score indicates that the subjects are more inclined to adopt positive coping styles, and a higher negative coping score indicates that the respondents are more willing to adopt negative coping styles. 
The patient's hope level was measured using the Chinese adapted version of the Herth hope index (HHI) (27). This scale includes 12 items in three dimensions (low hope level, median hope level, and high hope level). The four-point Likert scale method was adopted to assess the condition of participants in terms of a positive attitude toward reality and the future, taking positive actions, and maintaining close relationships with others. A higher score corresponded to a higher hope level. The Cronbach's coefficient $\alpha$ for the scale is 0.78 , and the test-retest reliability is 0.86 , showing good reliability and validity.

Personality traits refer to people's characteristic patterns of thoughts, feelings, and behaviors, which were evaluated using the Chinese adapted version of the Short Form of the NEO Five-Factor Inventory (NEO-FFI) (28). The NEO-FFI include five dimensions: neuroticism, extraversion, openness to experience, agreeableness, and conscientiousness (29). Each dimension includes 12 items. The five-point Likert scale method was adopted to calculate the total score for each dimension. The internal consistency Cronbach's $\alpha$ coefficient for the total scale is 0.74 , and the internal consistency Cronbach's $\alpha$ coefficient for the subscale is between 0.709 and 0.811 , indicating the good reliability and validity of the tool.

Self-esteem is an individual's subjective evaluation of their own worth. The level of self-esteem was measured using the Chinese adapted version of the Rosenberg self-esteem scale (RSES) $(30,31)$. The scale consists of 10 items, including five positive expressions and five negative expressions. The four-point Likert scale method was adopted to measure the overall self-esteem level of the patients. A higher total score on the scale reflected a higher level of self-esteem. The Cronbach's $\alpha$ coefficient for the scale is 0.88 , and the test-retest reliability is 0.85 , indicating the good reliability and validity of the scale.

\section{Data Analysis}

All data in this study were entered into a computer using Epidata software by two investigators. All statistical analyses were performed using SPSS 23.0 statistical software. The categorical variables were summarized as frequency and proportion, and the continuous variables were summarized by mean \pm standard deviation (SD).

We first compared the self-esteem score of the 157 included patients to a national normal model (23). Then, we applied $t$-test or analysis of variance (ANOVA) to check if the basic demographic characteristics of the patients were associated with their self-esteem level. Then, simple linear regression analysis was used to check the associations of the survey findings (e.g., loneliness, hope, coping styles, and personality traits) with self-esteem. Finally, we applied multiple regression analysis to explore the factors independently related to patients' self-esteem. Multiple stepwise regression analysis was performed using a stepwise method to screen independent variables (32). All tests were two-sided, and $P<0.05$ was considered statistically significant.

\section{RESULTS}

\section{Basic Characteristics of Participants}

The basic characteristics of the included patients were recorded from the completed questionnaires. Of the 157 participants, $56.7 \%(n=89)$ were male and $75.2 \%(n=118)$ were married. The mean age was $36.45 \pm 13.85$ years. The majority of the patients had acute non-lymphocytic leukemia $(75 / 157,47.8 \%)$ or acute lymphoblastic leukemia $(44 / 157,28.3 \%)$. Most of the included patients had a high school education, a low economic status, and medical insurance (Table 1).

\section{Self-Esteem Level of the Included Patients}

Among the 157 included patients with hematologic cancer, the mean self-esteem score was $26.86 \pm 2.34$, which was lower than the national norm $(28.75 \pm 4.86 ; P<0.01)(23)$. The results of comparisons of the basic demographic characteristics of the 157 patients according to their self-esteem scores are presented in Table 1. The self-esteem level of low-income patients was significantly lower than that of high-income patients $(P<0.01)$. Additionally, rural patients' self-esteem level was significantly lower than that of urban patients $(P<0.01)$.

Simple linear regression analysis was performed to identify factors associated with patients' self-esteem (Table 2). Coping style, hope, five personality traits, and loneliness were found to be associated significantly with patients' self-esteem level $(P<0.01)$.

The multiple linear regression analysis results for factors independently associated with patients' self-esteem are present in Table 2. Maintaining close relationships with others, conscientiousness, extraversion, agreeableness, active coping style, number of hospitalizations, payment method for medical expenses, and length of illness were found to be independently associated with the self-esteem of hematological cancer patients.

\section{DISCUSSION}

In the present cross-sectional study, the mean self-esteem score of patients with hematologic malignancies was $26.86 \pm 2.34$, which is significantly lower than a previously reported normal score for the national population of China (31). The occurrence of a malignant tumor is a major negative life event (33). The diagnosis of a malignant tumor and the pain of the cancer itself constitute a strong reminder of the possibility of death, easily leading to psychological problems such as depression and death anxiety in patients (34). The low level of the self-esteem among the patients included in the present study may be due to the lack of health education about their disease and misunderstanding of their disease progression and treatment. According to a survey study, blood disease is a risk factor for death anxiety, and good self-esteem can effectively alleviate death anxiety (35). According to the results of Yang et al., a certain amount of psychological counseling based on conventional treatment can enhance the confidence of patients with hematological malignancies that they will be cured, thereby improving their quality of life (36).

In the present study, evaluation of the individual demographic characteristics of the included patients showed that with more or longer hospitalization, self-esteem decreased $(P<0.001)$. 
TABLE 1 | Associations between demographic characteristic and self-esteem among the 157 included patients with hematological cancers.

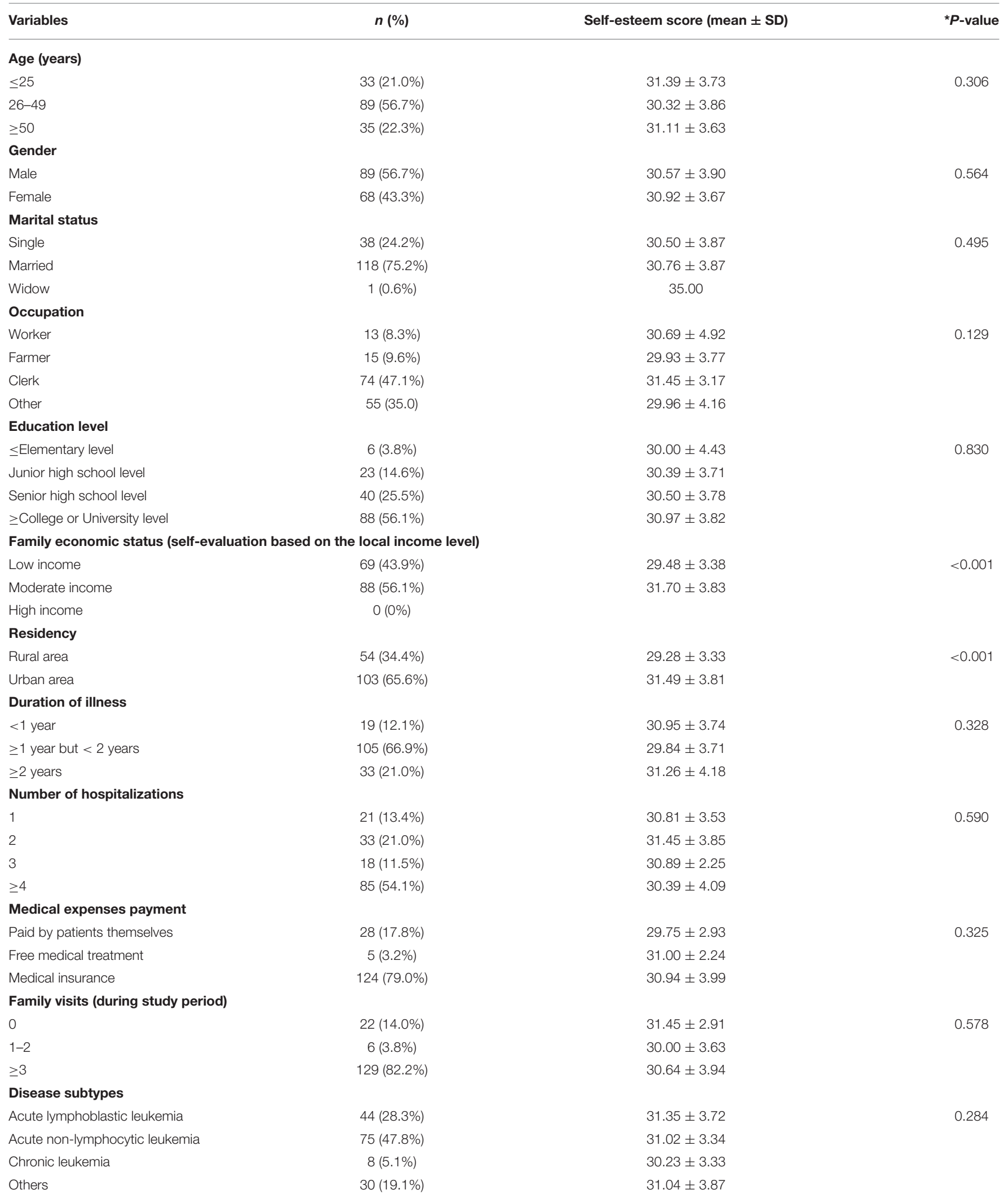

${ }^{*}$-value from $t$-test or ANOVA for associations of the self-esteem scores according to each demographic characteristic. 
TABLE 2 | Linear regression results for factors associated with patient self-esteem $(n=157)$.

\begin{tabular}{|c|c|c|c|c|}
\hline Independent variables & Beta & $95 \%$ confidence interval & $\boldsymbol{F}$ & $P$-value \\
\hline \multicolumn{5}{|l|}{ Simple linear regression } \\
\hline Loneliness scale & 0.500 & & 51.539 & $<0.001$ \\
\hline Hope level & 0.551 & & 67.592 & $<0.001$ \\
\hline Positive attitude toward reality and the future & 0.430 & & 35.066 & $<0.001$ \\
\hline Active coping styles & 0.408 & & 30.900 & $<0.001$ \\
\hline Maintaining close relationships with others & 0.617 & & 95.048 & $<0.001$ \\
\hline Positive coping style & 0.444 & & 38.094 & $<0.001$ \\
\hline Negative coping style & -0.220 & & 7.889 & 0.006 \\
\hline Neuroticism & -0.454 & & 40.137 & $<0.001$ \\
\hline Extraversion & 0.534 & & 61.845 & $<0.001$ \\
\hline Openness to experience & 0.180 & & 5.215 & 0.024 \\
\hline Agreeableness & 0.443 & & 37.867 & $<0.001$ \\
\hline Conscientiousness & 0.536 & & 62.546 & $<0.001$ \\
\hline \multicolumn{5}{|l|}{ Multiple linear regression } \\
\hline Active emotion-focused coping style & 0.491 & {$[0.871,1.538]$} & 31.862 & $<0.001$ \\
\hline Conscientiousness & 0.402 & {$[0.183,0.367]$} & & $<0.001$ \\
\hline Extraversion & 0.273 & {$[0.114,0.312]$} & & $<0.001$ \\
\hline Number of hospitalizations & -0.219 & {$[-1.086,-0.373]$} & & $<0.001$ \\
\hline Medical expenses payment & -0.196 & {$[-1.517,-0.406]$} & & 0.001 \\
\hline Agreeableness & -0.239 & {$[-0.363,-0.073]$} & & 0.004 \\
\hline Positive coping style & 0.166 & {$[0.355,2.530]$} & & 0.010 \\
\hline Duration of illness & -0.113 & {$[-1.179,-0.045]$} & & 0.035 \\
\hline
\end{tabular}

Beta is Standardized Coefficient. Adjusted R Square is 0.613. $P<0.01$.

Hope is a multi-dimensional psychological force that can motivate patients to adopt a positive attitude and help them overcome anxiety, fear, and other bad emotions during treatment (37). The present study showed that maintaining close relationships with others was associated with a better level of self-esteem than not maintaining close relationships $(P<$ 0.001). Maintaining an intimate relationship with others helps patients obtain sufficient social support, including mental or material help from family, friends, etc., which can buffer stress and maintain a good emotional experience (38). Zhao et al. reported that the social support of patients with hematological disorders is negatively correlated with anxiety and depression. The more social support patients receive, the less negative emotions such as anxiety and depression they will experience, and the higher their level of self-esteem will be (39). Good social support can improve the self-esteem of patients with hematological tumors, reduce their psychological pressure, and promote improvement of their disease (40). Li et al. pointed out that the combinations of cultural and sports activities and personalized health education can effectively improve patients' ability to maintain intimate relationships with others and increase the self-esteem of patients (41). Therefore, in practice, we recommend efforts to achieve good communication between patients with hematologic malignancies and their doctors and to encourage good emotional support from family members to directly improve the level at which the patient maintains close relationships with others, thereby increasing their selfesteem level.
A positive coping style can improve people's mood and improve their ability to deal with issues. On the other hand, a negative coping style makes an individual gradually sink, unwilling to accept facts, which damages the patient's physical and mental health and is not conducive to later recovery and treatment $(42,43)$. The results of the present study suggest that the active coping style of patients is an important factor associated with the self-esteem level of hematological cancer patients $(P=0.01)$. People with high self-esteem are more inclined to adopt active coping methods such as problem-solving and help, while those with low self-esteem are more inclined to adopt negative coping styles such as self-blame, fantasies, and avoidance (44). Conversely, adopting positive coping styles can enable individuals to get more social support and help, which is conducive to problem-solving, stress relief, and higher life satisfaction (45). Therefore, in practice, when patients are faced with setbacks, encouraging and guiding them to adopt positive coping styles is conducive to the individual's good adaptation to the event, prompting patients to make more positive evaluations of their own condition and experience higher life satisfaction.

Loneliness is a psychological state in which the individuals are dissatisfied with their interpersonal relationship status, and it is often accompanied by negative emotional reactions such as helplessness and depression (46). The unbearable sense of mental loss hurts cognition and behavior and is an important risk factor for mental and physical health problems (6). Loneliness has been shown in several studies to be negatively associated with recovery from a number of chronic diseases, particularly 
cancer. Additionally, loneliness increases the risk of physical and mental health problems and increases premature mortality and national mortality. It was reported that loneliness affects patients' self-esteem through feelings such as helplessness and depression $(47,48)$. In the present study, loneliness was negatively associated with patients' self-esteem on simple linear regression analysis, but not on the multiple linear regression analysis. The reason is unknown, and thus, further investigation and analysis on this topic is warranted.

The five personality traits are human beings' relative stability traits, which maintain a relatively stable worrying tendency or worrying habits with individual differences at different times and in different situations (49). Conversely, when facing the same pressure, individuals with low self-esteem attribute the event to self-ability limitations, and individuals with high self-esteem attribute the event to external causes $(50,51)$. The present study shows that conscientiousness, extraversion, and agreeableness are factors associated with the self-esteem of hematological cancer patients. Personality traits are relatively stable over time and have cross-contextual stability. However, such characteristics vary from person to person, which is responsible for the differences in personality between people. Self-esteem refers to the evaluation made or usually held by individuals. It is a spiritual need, the core of personality, and the core of mental health. Therefore, each person's personality is different and stable, which means that the level of self-esteem also varies from person to person and cannot be adjusted (52).

To our best knowledge, this is the first study to explore the factors associated with the self-esteem of patients with hematologic malignancies. There are several limitations for the present study. First, as the present study is a cross-sectional study, not a cohort study, we cannot conclude that the factors we found associated with patients' self-esteem are independent influencers. Next, in the present study, we included patients both at the beginning of their diagnosis stages and who were receiving treatment with chemotherapy/radiotherapy to capture any possible mental features in this unique group. A subgroup analysis of patients in different treatment stages will be performed soon. Finally, the included patients came from a tertiary hospital in Beijing, which resulted in the patients having high education

\section{REFERENCES}

1. Taylor J, Xiao W. Diagnosis and classification of hematologic malignancies on the basis of genetics. Blood. (2017) 130:410-23. doi: 10.1182/blood-2017-02734541

2. Zomerdijk N, Turner JM. Adult-related haematopoietic stem cell donor experiences and the provision of information and psychosocial support: a systematic literature review. Eur J Cancer Care. (2019) 28:e12932. doi: 10.1111/ecc.12 932

3. Wang N, Feng YJ, Wang BH, Fang LW, Cong S, Li YC, et al. [Disease burden of leukemia in the Chinese population, in 1990 and 2013]. Zhonghua Liu Xing Bing Xue Za Zhi. (2016) 37:783-7. doi: 10.3760/cma.j.issn.0254-6450.2016. 06.008

4. Zhao P, Dai M, Chen W, Li N. Cancer trends in China. Jpn J Clin Oncol. (2010) 40:281-5. doi: 10.1093/jjco/hyp187 levels. Because Beijing is the capital of China, there are many people with high education qualifications. Additionally, Beijing attracts many people with high education qualifications seeking medical treatment. The scope of the study will be expanded in later research efforts, and the results of the study will be optimized. Further research with a cohort design or even a randomized controlled design is needed to confirm our findings.

\section{CONCLUSION}

Patients with hematologic malignancies experience low selfesteem. Factors, including maintaining close relationship with others, conscientiousness, extraversion, agreeableness, and active coping style, were found to be independently associated with the self-esteem level of patients with hematologic malignancies. For patients with hematologic malignancies, health providers should apply patient-centered and targeted interventions to improve patients' self-esteem, reduce adverse psychological emotions, promote overall well-being, and improve their quality of life.

\section{DATA AVAILABILITY STATEMENT}

The raw data supporting the conclusions of this article will be made available by the authors, without undue reservation.

\section{ETHICS STATEMENT}

The studies involving human participants were reviewed and approved by The Ethics Committee of the Space Center Hospital approved the study (approval \# 20200108-BSYJS-01). The patients/participants provided their written informed consent to participate in this study.

\section{AUTHOR CONTRIBUTIONS}

JY designed the study and revised the manuscript. HD and QW conducted all the data analyses and wrote the manuscript. YY collected the data. HP provided guidance and assistance in the topic selection design and implementation, and thesis writing process. All authors read and approved the final manuscript.
5. Parvan K, Jabbarzadeh Tabrizi F, Rahmani A, Ghojazadeh M, Azadi A, Golchin M. The relationship between hope and self-esteem in patients with leukemia. J Caring Sci. (2015) 4:217-23. doi: 10.15171/jcs.20 15.022

6. Moussas GI, Papadopoulou AG, Christodoulaki AG, Karkanias AP. [Psychological and psychiatric problems in cancer patients: relationship to the localization of the disease]. Psychiatriki. (2012) 23:46-60.

7. Guan J. An investigation and study of psychological health condition of patient with blood disease. Health Psychol J. (2003) 11:5-7.

8. Sowislo JF, Orth U. Does low self-esteem predict depression and anxiety? A meta-analysis of longitudinal studies. Psychol Bull. (2013) 139:21340. doi: $10.1037 / \mathrm{a} 0028931$

9. Chang XD. Influence of holistic nursing on psychology and compliance of patients with hematological diseases chemotherapy. Cardiovasc Dis J Integr Tradit Chin West Med. (2016) 4:115-8. 
10. Wang YN. Identity reconciler theory: a new indigenous self-esteem theory. Psychol Technol Appl. (2020) 8:185-92.

11. Harpin V, Mazzone L, Raynaud JP, Kahle J, Hodgkins P. Long-term outcomes of ADHD: a systematic review of self-esteem and social function. J Atten Disord. (2016) 20:295-305. doi: 10.1177/10870547134 86516

12. Hogg MA, Vaughan GM. Social Psychology. vol. 6. New York: NY Pearson Prentice Hall (2011).

13. Ichimura-Abe M. [Recovery behavior for decreased self-esteem related to the level and stability of self-esteem]. Shinrigaku Kenkyu. (2011) 82:3629. doi: $10.4992 /$ jjpsy. 82.362

14. Tomasiewicz AK. Body image and self-esteem of patients with oncohematological diseases after chemotherapy. Psychoterapia. (2015) 172:73-84. doi: 10.12740/PT/31993

15. Corrigan PW, Kerr A, Knudsen L. The stigma of mental illness: explanatory models and methods for change. Appl Prev Psychol. (2005) 11:17990. doi: 10.1016/j.appsy.2005.07.001

16. Gheihman G, Zimmermann C, Deckert A, Fitzgerald P, Mischitelle A, Rydall A, et al. Depression and hopelessness in patients with acute leukemia: the psychological impact of an acute and lifethreatening disorder. Psychooncology. (2016) 25:979-89. doi: 10.1002/po n. 3940

17. Yilmaz O, Zehra B, Torun YA, Karakukcu M. Anxiety, depression, self-esteem, and attitude toward illness among children with leukemia and lymphoma in remission. HemaSphere. (2019) 3:771-2. doi: 10.1097/01.HS9.0000564924.99 794.1f

18. Tremolada M, Taverna L, Bonichini S, Basso G, Pillon M. Self-esteem and academic difficulties in preadolescents and adolescents healed from paediatric leukaemia. Cancers. (2017) 9:5. doi: 10.3390/cancers90 60055

19. Khrushchev S, Vybornykh D, Tkhostov A, Pluzhnikov I, Savchenko V. Psychological disturbances and perception of disease in patients with hematological malignancies. Psycho Oncol. (2017) 26:75-6.

20. Kim M, Nam S, Kim J. The effects of sociodemographic factors, cancer-related factors, and self-esteem on self-reported worries of childhood cancer survivors in Korea: P1-31. Pscyho Oncol. (2016) 25.

21. von Elm E, Altman DG, Egger M, Pocock SJ, Gøtzsche PC, Vandenbroucke JP. The strengthening the reporting of observational studies in epidemiology (STROBE) statement: guidelines for reporting observational studies. Ann Intern Med. (2007) 147:573-7. doi: 10.7326/0003-4819-147-8-20071016000010

22. Sharma SK, Mudgal SK, Thakur K, Gaur R. How to calculate sample size for observational and experimental nursing research studies? Natl J Physiol Pharm Pharmacol. (2020) 10:1-8. doi: 10.5455/njppp.2020.10.0930717 102019

23. Qian M. A Study on the Goal Level and Self-Evaluation of Depressed People. Beijing: Peking University (1995).

24. Russell D, Peplau LA, Cutrona CE. The revised UCLA Loneliness Scale: concurrent and discriminant validity evidence. $J$ Pers Soc Psychol. (1980) 39:472-80. doi: 10.1037/0022-3514.3 9.3.472

25. Wang DW, Zhang JX. Factor analysis of the simplified coping style questionnaire. J Shandong Univ. (2014) 52:96-100. doi: 10.6040/j.issn.1671-7554.0.2013.380

26. Yaning X. On the Reliability and Validity of the Simple Coping Style Scale. Chin J Clin Psychol. (1998) 6:53-4.

27. Zhao HP, Wang J. Social Support and Hope in Hemodialysis Patients. Chiang Mai: Chiang Mai University (2000).

28. Costa Jr P, McCrae R. Professional manual: Revised NEO Personality Inventory (NEO-PI-R) and NEO Five-Factor Inventory (NEO-FFI) Professional Manual. Odessa, FL: Psychological Assessment Resources (1992).

29. Rosellini AJ, Brown TA. The NEO Five-Factor Inventory: latent structure and relationships with dimensions of anxiety and depressive disorders in a large clinical sample. Assessment. (2011) 18:27-38. doi: 10.1177/10731911103 82848
30. Rosenberg M. Society and the Adolescent Self-Image. Princeton, NJ: Princeton UP. (1965).

31. Dai X. Handbook of Common Psychological Assessment Scales. Beijing: People’s Military Medical Publishing House (2010). p. 310-3.

32. Heckler CE. Applied multivariate statistical analysis. Technometrics. (2005) 47:517. doi: 10.1198/tech.2005.s319

33. Salleh MR. Life event, stress and illness. Malays J Med Sci. (2008) 15:9-18. doi: 10.1016/0021-9169(79)90015-1

34. Hu CW, Ding N, Ding CL, Cao YH. A Preliminary study on death anxiety of hospitalized tumor patients and its influencing factors. Chin J Clin Healthcare. (2014) 17:320-1. doi: 10.3969/J.issn.1672-6790.2014. 03.037

35. Na D, Yan T, Chengwen H, Shujuan C, Zhengqin H, Chunfeng $\mathrm{W}$, et al. Relationship between death anxiety and selfesteem in patients with malignant tumors. Chinese $J$ Pract Nurs. (2015) 29:2246-9. doi: 10.3760/cma.j.issn.1672-7088.2015. 29.020

36. Yang JM, Chen J. Mental status and treatment of patients with malignant hematological diseases. Clin Blood J. (2004) 17:319-20. doi: 10.3969/j.issn.1004-2806.2004.06.004

37. Guindon $\mathrm{MH}$. Toward accountability in the use of the self-esteem construct. J Couns Dev. (2002) 80:204-14. doi: 10.1002/j.1556-6678.2002. tb00184.x

38. Davey-Rothwell MA, Stewart J, Vadnais A, Braxton SA, Latkin CA. The role of partner support among women with depressive symptoms. Commun Ment Health J. (2017) 53:415-9. doi: 10.1007/s10597-0170101-3

39. Zhao ML, Cao FL. Correlation analysis of anxiety, depression and psychological resilience and social support in patients with hematological diseases. People's Liberation Army Nurs J. (2018) 35:8-12. doi: $\quad 10.3969 /$ j.issn.1008-9993.2018. 08.002

40. Karaca A, Yildirim N, Cangur S, Acikgoz F, Akkus D. Relationship between mental health of nursing students and coping, self-esteem and social support. Nurs Educ Today. (2019) 76:44-50. doi: 10.1016/j.nedt.201 9.01.029

41. Li T, Wang JX, Chang MM, Zhang B. Individualized health education combined with cultural and sports activities to intervene the effect of colostomy. Chin J Health Psychol. (2020) 28:1166-70. doi: 10.13342/j.cnki.cjhp.2020. 08.011

42. Chen SK. The Guidance for Clinical Psychology and Nursing. Beijing: Beijing Science and Technology Press (2002).

43. Emmanuel EN, Creedy DK, St John W, Brown C. Maternal role development: the impact of maternal distress and social support following childbirth. Midwifery. (2011) 27:265-72. doi: 10.1016/j.midw.2009. 07.003

44. Xu ZY, Zu S, Xiang YT, Wang N, Guo ZH, Kilbourne AM, et al. Associations of self-esteem, dysfunctional beliefs and coping style with depression in patients with schizophrenia: a preliminary survey. Psychiatry Res. (2013) 209:340-5. doi: 10.1016/j.psychres.2013. 02.012

45. Cen YY, Zheng X. Research on the Correlation between college students' self-esteem and coping styles. Chin $J$ Clin Psychol. (2005) 13:167-9. doi: 10.3969/j.issn.1005-3611.2005. 02.014

46. Hyland P, Shevlin M, Cloitre M, Karatzias T, Vallières F, McGinty G, et al. Quality not quantity: loneliness subtypes, psychological trauma, and mental health in the US adult population. Soc Psychiatry Psychiatr Epidemiol. (2019) 54:1089-99. doi: 10.1007/s00127-018-1597-8

47. Deckx L, van den Akker M. A systematic literature review on the association between loneliness and coping strategies. Psychol Health Med. (2018) 23:899-916. doi: 10.1080/13548506.2018.14 46096

48. Shah SGS, Nogueras D, van Woerden H, Kiparoglou V. Effectiveness of digital technology interventions to reduce loneliness in adults: a protocol for a systematic review and meta-analysis. 
BMJ Open. (2019) 9:e032455. doi: 10.1136/bmjopen-2019-0 32455

49. Zheng Z, Wang FS. Incidence and influencing factors of subclinical somatic deformity in college students. In: Abstracts of the 21st National Conference on Psychology. Beijing (2018).

50. Wang Y, Yang J. The role of personality traits in regulating individual psychological stress response. $A d v$ Psychol Sci. (2015) 23:1453-60. doi: 10.3724/SP.J.1042.2015. 01453

51. Wang YY, Sun HW, Liu JH, Gao SH, Wang EG, Xu N. Self-esteem, personality characteristics and emotional status of prisoners for duty crimes. Chin Ment Health J. (2012) 26:393-8. doi: 10.3969/j.issn.1000-6729.2012. 05.016

52. Pan YQ. A comparative study of personality traits and selfesteem of music and non-music majors. In: Shanghai Conservatory of Music Proceedings of the 3rd Chinese Musicians Association Symposium of Music Psychology Society. Shanghai (2008). p. 436-527.
Conflict of Interest: The authors declare that the research was conducted in the absence of any commercial or financial relationships that could be construed as a potential conflict of interest.

Publisher's Note: All claims expressed in this article are solely those of the authors and do not necessarily represent those of their affiliated organizations, or those of the publisher, the editors and the reviewers. Any product that may be evaluated in this article, or claim that may be made by its manufacturer, is not guaranteed or endorsed by the publisher.

Copyright (C) 2021 Yu, Dong, Wu, Yang and Pi. This is an open-access article distributed under the terms of the Creative Commons Attribution License (CC BY).

The use, distribution or reproduction in other forums is permitted, provided the original author(s) and the copyright owner(s) are credited and that the original publication in this journal is cited, in accordance with accepted academic practice. No use, distribution or reproduction is permitted which does not comply with these terms. 